\title{
Simulating the Effect of a Class of Sensor Fuzed Munitions for Artillery on a Multiple Target Element System
}

\author{
Henri Kumpulainen Bernt M. Åkesson \\ Information Technology Division, Finnish Defence Research Agency, Finland, bernt .akesson@mil.fi
}

\begin{abstract}
This paper presents a method for analyzing the effect of a class of artillery-launched sensor fuzed munitions on a target, which is a system consisting of several target elements and a fault logic. The target elements are armored vehicles and the munitions are designed specifically to attack single vehicles. We consider munitions which may contain one or two submunitions. We want to address the following questions: what is the probability of disabling the system given the number of ammunition, and similarly, how much ammunition is needed for disabling the system with a given confidence level. The proposed method is based on Markov chains rather than Monte Carlo simulation.
\end{abstract}

Keywords: Markov processes, probability, set theory, operations research, mathematical model, algorithms, weapons

\section{Introduction}

The term sensor fuzed munition is here used to denote munitions with target recognition capability and the ability to autonomously search for, detect, recognize and attack single target elements with specific signatures. Sensor fuzed munitions are generally intended for use against armored vehicles. Sensor fuzed munitions engage their targets from above from a distance using explosively formed projectiles (Dullum, 2008).

In this paper we consider sensor fuzed submunitions that are delivered by an artillery projectile. The projectile travels on a ballistic trajectory and ejects one or two sensor fuzed submunitions over a desired release point using a time fuze. The submunitions operate independently of each other, and after stabilizing and retarding their descent, scan the area beneath them for suitable target elements. Figure 1 illustrates the operation of the munitions.

A target consists of a number of target elements in a formation. Defeat of the target is defined by the damaging of specific combinations of target elements.

We propose an approach based on Markov chains. This approach has the benefit that once the effect of a single projectile has been calculated, the effects of further projectiles can be calculated by simple matrix operations.

Analytical models for different types of sensor fuzed munitions have been derived by (Halsør and Kvifte, 2003). This paper extends that work.

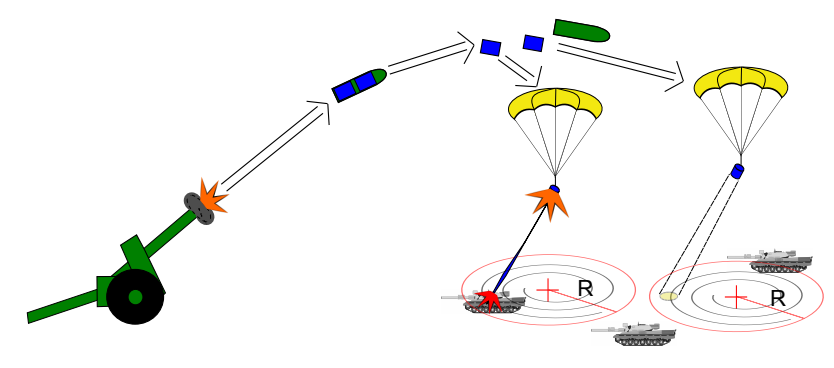

Figure 1. Schematic of the firing of an artillery projectile containing two sensor fuzed anti-tank submunitions.

A corresponding method as in this paper has been previously applied to fragmenting munitions by (Pettersson et al., 2011). The main difference is that a single fragmenting munition can damage all target elements in the area, whereas a sensor fuzed submunition can only damage a single target element.

An alternative approach that has been used before for this type of problems is to apply Monte Carlo methods, as outlined in e.g. (Halsør and Kvifte, 2003) and (NATO Standardization Office, 2012). Monte Carlo methods are known to be computationally expensive when we need accurate estimates of the kill probabilities, since their error is proportionate to $1 / \sqrt{n}$ where $n$ is the number of replications.

The paper is outlined as follows. First, an overview of the method is given. After this, a method for computing the probabilities of encountering different target element subsets is presented. Then the probabilities in the cases with one and two submunitions are derived. Finally, the failure probability of a system of target elements is considered.

\section{Overview of the Method}

An illustration of the method is provided in Figure 2. The computations are performed in three steps. First, the probabilities of a submunition encountering each subset of target elements are computed. After this, a state transition matrix is constructed and finally, the effect of a given number of munitions is computed. The state vector can be used together with fault logics to determine the probability of the target being broken.

The following assumptions are made:

1. The position of each target element is known 


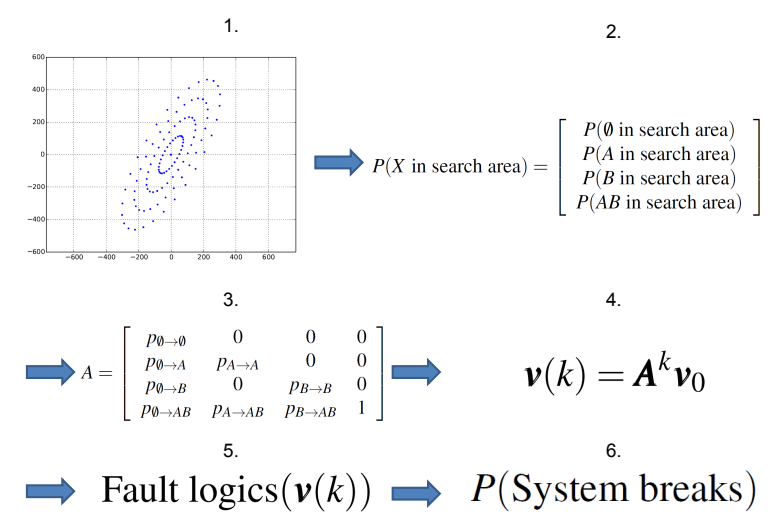

Figure 2. Illustration of the method.

\section{The target elements are identical}

3. A single submunition can only kill one target element

4. A target element has two states, functional and broken

5. Broken target elements are never attacked

6. The sensor is scanning, seeing only part of the search area at a time, and the munition will attack the first target it detects that fits given criteria. See Figure 1.

7. The search area, also known as the sensor footprint, is circular in shape

8. The target elements are stationary

The second assumption can be generalized by having different probabilities for detection, hit and kill for different target element types. The seventh assumption can be easily relaxed to other footprint shapes.

The carrier projectile is assumed to follow a ballistic trajectory, which can be computed using, e.g., a modified point-mass model. In this paper, the release point is for simplicity assumed to follow a bivariate normal distribution on the ground plane. The mean and standard deviations are assumed to be known.

A sensor fuzed submunition is characterized by the following parameters:

- Radius $R$ of the search area

- Reliability $p_{\mathrm{f}}$

- Detection probability $p_{\mathrm{d}}$

- Hit probability $p_{\mathrm{h}}$

- Kill probability $p_{\mathrm{k} \mid \mathrm{h}}$, i.e., the probability of the target element breaking when hit
The probability of detection and the probability of kill may depend of the target element type together with weather and terrain conditions. The probability of detection may also depend on the distance from the center of the search area. In this paper the scanning method of the submunition is not specified, and thus all target elements inside the search area have equal probability of being detected first.

Some parameter values for existing sensor fuzed munitions can be found in open sources. For example, for a certain munition, (Dullum, 2008) reports a search area radius $R=100 \mathrm{~m}$, and (Kosola and Solante, 2013) report a hit probability of $p_{\mathrm{h}}=0.8$ and a kill probability of $p_{\mathrm{k} \mid \mathrm{h}}=0.95$.

\section{Computing the Encounter Probabil- ities}

We consider $n$ identical target elements in the target area. Let us denote the set of target elements by $T=$ $\left\{T_{1}, T_{2}, T_{3}, \ldots, T_{n}\right\}$, and the probability that the set of broken target elements after $k$ artillery rounds is exactly $X$ by $P_{k}(X)$.

Given a position $(x, y)$ on the ground plane, there is a probability $p(x, y)$ of a sensor fuzed submunition starting its search in that point. The probability is directly the probability density function of the bivariate normal distribution. We look at a circular area with radius $R$ around $(x, y)$ and select all target elements within it. This corresponds to subset $X_{i} \subseteq T$. The integrand function returns a vector with $\operatorname{dim}(\boldsymbol{v})=2^{n}$, whose $i$ th element has value $p(x, y)$.

Let $\boldsymbol{P}$ (set in search area) be a vector of length $2^{n}$ containing the probabilities that a single round encounters a given subset of the target elements within its search area,

$\boldsymbol{P}($ set in search area $)=\left[\begin{array}{c}P(\emptyset \text { in search area }) \\ P\left(\left\{T_{1}\right\} \text { in search area }\right) \\ P\left(\left\{T_{2}\right\} \text { in search area }\right) \\ P\left(\left\{T_{1}, T_{2}\right\} \text { in search area }\right) \\ \vdots \\ P\left(\left\{T_{1}, T_{2}, \ldots, T_{n}\right\} \text { in search area }\right)\end{array}\right]$

One approach, proposed in (Halsør and Kvifte, 2003), is to integrate over an area to obtain the probabilities of encountering each target element combination,

$$
\boldsymbol{P}(\text { set in search area })=\int_{-\infty}^{\infty} \int_{-\infty}^{\infty} \boldsymbol{f}(x, y) \mathrm{d} x \mathrm{~d} y
$$

where the integrand $\boldsymbol{f}(x, y)$ is a vector-valued function, such that for $i=1, \ldots, 2^{n}$

$$
f_{i}(x, y)= \begin{cases}p(x, y), & \text { if subset } X_{i} \text { is within distance } R \\ 0, & \text { from point }(x, y) \\ \text { otherwise }\end{cases}
$$


Table 1. Encounter probabilities corresponding to the situation in Figure 3

\begin{tabular}{ll}
\hline Set $X$ & $P(X$ in search area $)$ \\
\hline$\emptyset$ & 0.0138 \\
$\{A\}$ & 0.0018 \\
$\{B\}$ & 0.0781 \\
$\{A, B\}$ & 0.1427 \\
$\{C\}$ & 0.1834 \\
$\{A, C\}$ & 0.1521 \\
$\{B, C\}$ & 0.0426 \\
$\{A, B, C\}$ & 0.3853 \\
\hline
\end{tabular}

where $p(x, y)$ is the value of the probability density function at point $(x, y)$.

\subsection{Example}

The integral in (2) can be performed for an elliptical area centered on the aimpoint. Figure 3 illustrates the situation. The dispersion pattern is shown as ellipses. Three example functioning points are shown. At functioning point $\mathrm{P}_{1}$ only target element $\mathrm{B}$ can be detected, at point $\mathrm{P}_{2} \mathrm{~A}$ and $\mathrm{C}$ can be detected and at point $\mathrm{P}_{3}$ all three target elements can be detected. When integrating over the area using (2) we obtain the encounter probabilities listed in Table 1.

\section{State Transition Matrix}

Let $P_{k}(X)$ be the probability that the set of target elements $X$ is broken at time step $k$. Let $\boldsymbol{v}(k)$ be a vector that contains the values of $P_{k}(X)$ for every subset $X \subseteq T$ in bit order, i.e., $\boldsymbol{v}(k)=$ $\left[P_{k}(\emptyset), P_{k}\left(\left\{T_{1}\right\}\right), P_{k}\left(\left\{T_{2}\right\}\right), P_{k}\left(\left\{T_{1}, T_{2}\right\}\right), \ldots\right]^{T}$, at time step $k$.

We can interpret the system as a state machine where a state is characterized by the set of broken target elements and during each time step exactly one round of the weapon system is fired. The vector $\boldsymbol{v}(k)$ contains the state probabilities. Initially, all target elements are functional, thus $\boldsymbol{v}(0)=[1,0, \ldots, 0]^{T}$.

We can compute a state transition matrix $\boldsymbol{A}$ so that, for all $k$,

$$
\boldsymbol{v}(k+1)=\boldsymbol{A} \boldsymbol{v}(k) .
$$

After firing $k$ rounds the end state is given by

$$
\boldsymbol{v}(k)=\boldsymbol{A}^{k} \boldsymbol{v}(0) \text {. }
$$

The state transition matrix $\boldsymbol{A}$ is defined as

$$
\boldsymbol{A}=\left[P\left(X_{j} \rightarrow X_{i}\right)\right]_{1 \leq i, j \leq 2^{n}}
$$

where $P\left(X_{j} \rightarrow X_{i}\right)$ is the probability of moving from state $X_{j} \subseteq T$ to state $X_{i} \subseteq T$.

Matrix $\boldsymbol{A}$ is rather large and has dimensions $2^{n} \times 2^{n}$. However, $\boldsymbol{A}$ is rather sparse, which means that all matrix operations will be faster when implemented using sparse matrices.
During an artillery firing an individual target element can either remain functional or break but broken target elements can never return back to functional. This means that $P\left(X_{j} \rightarrow X_{i}\right)=0$ if $X_{j} \nsubseteq X_{i}$, and thus $P\left(X_{j} \rightarrow X_{i}\right)$ can only be nonzero if $X_{j} \subseteq X_{i}$. In (Pettersson et al., 2011) it was shown that when a single round is capable of killing all target elements the number of nonzero elements in $\boldsymbol{A}$ is at most $3^{n}$.

If, on the other hand, at most $n_{K}$ target elements can be killed by a single round, then $P\left(X_{j} \rightarrow X_{i}\right)$ can only be nonzero if $\left|X_{i} \backslash X_{j}\right| \leq n_{K}$ and $X_{j} \cap X_{i}=X_{j}$. The number of nonzero elements matrix $\boldsymbol{A}$ is then

$$
\begin{aligned}
& N(\text { nonzero elements in } \boldsymbol{A})= \\
& \qquad \sum_{j=1}^{2^{n}} N(\text { nonzero elements in column } j \text { of } \boldsymbol{A}) \leq \\
& \qquad \sum_{i=0}^{n}\left(\begin{array}{c}
n \\
i
\end{array}\right) \sum_{m=0}^{n_{K}}\left(\begin{array}{c}
n-i \\
m
\end{array}\right) .
\end{aligned}
$$

If one target element can be killed we have at most $(n+$ 2) $2^{n-1}$ nonzero elements and if two target elements can be killed the number of nonzero elements is at most $\left(n^{2}+\right.$ $3 n+8) 2^{n-3}$.

\subsection{Projectile with a Single Sensor Fuzed Sub- munition}

If the encountered set of target elements is $X_{k}$ and the initial state is $X_{j}$, the set of killed target elements in the encountered set is $X_{j} \cap X_{k}$. The set of functional target elements in the encountered set is $X_{k} \backslash X_{j}$.

Since the target elements are assumed identical, for any target element $X \in X_{k}$ we have that

$$
P\left(X \text { killed } \mid X_{k} \text { in search area and } X_{j} \cap X_{k} \text { killed }\right)=
$$$$
P\left(1 /\left|X_{k} \backslash X_{j}\right| \text { killed }\right) \text {. }
$$

The probability of killing exactly one target element out of $m$ is

$$
P(1 / m \text { killed })=\left(1-\left(1-p_{\mathrm{d}}\right)^{m}\right) p_{\mathrm{h}} p_{\mathrm{k} \mid \mathrm{h}} p_{\mathrm{f}} / m .
$$

When only one target element can be killed each time step, the exact probability of moving from state $X_{j} \subseteq T$ to state $X_{i} \subseteq T$ in one time step is

$$
\begin{aligned}
& P\left(X_{j} \rightarrow X_{i}\right)=P\left(X_{j} \rightarrow\left(X_{j} \cup\left\{T_{i}\right\}\right)\right)= \\
& \sum_{\substack{X_{k} \subseteq T, \\
\text { s.t. }}} P\left(X_{k} \text { in search area }\right) P\left(1 /\left|X_{k} \backslash X_{j}\right| \text { killed }\right) .
\end{aligned}
$$

Next we present an algorithm for calculating the state transition matrix more efficiently than the brute force approach of applying (10) for each nonzero element in the matrix. 

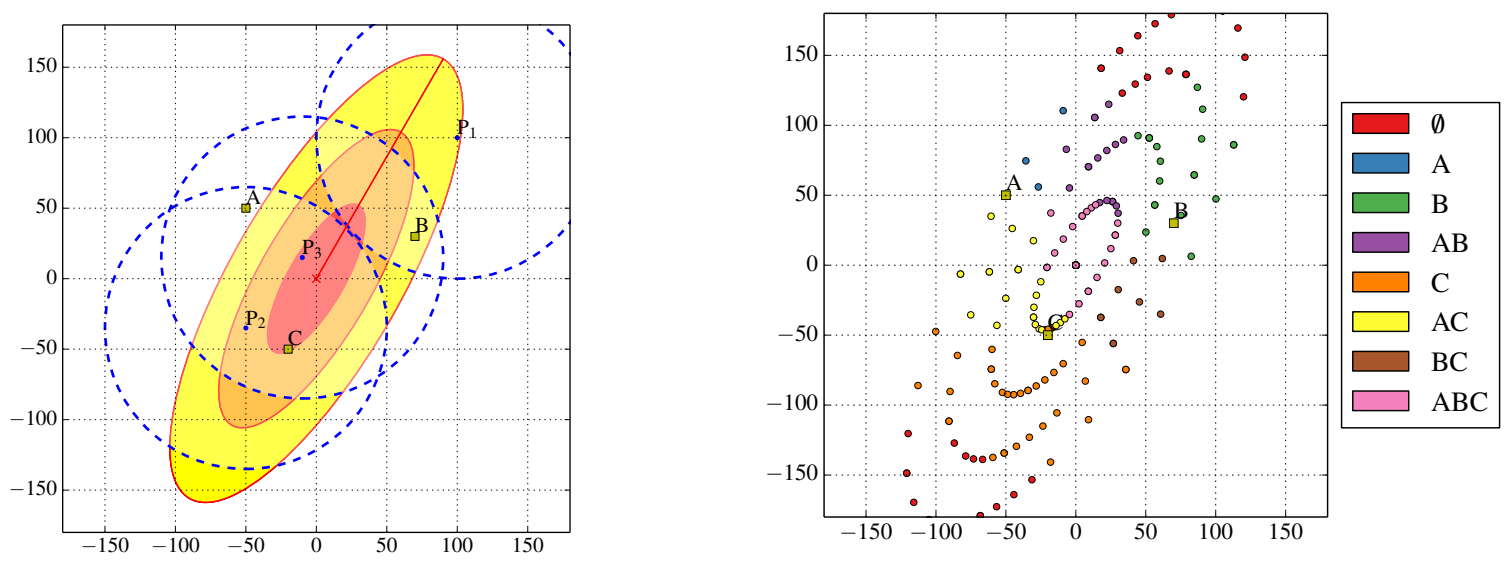

Figure 3. The figure on the left shows the positions of three target elements: A, B and C. The ballistic dispersion of the carrier projectile is marked by ellipses for one, two and three standard deviations. A single submunition is assumed. Three example functioning points $\mathrm{P}_{1}, \mathrm{P}_{2}$ and $\mathrm{P}_{3}$ have been plotted, with the sensor footprints outlined with dashed circles. The figure on the right shows the subsets found in each integration point.

Let us define $Q(X)$ as the probability that $X$ is a subset of the set of target elements inside the search area. We can calculate this probability as

$$
Q(X)=\sum_{\substack{Y \subseteq T \\ \text { s.t. } X \subseteq Y}} P(Y \text { in search area }) .
$$

The probability that a set of target elements $X$ is inside the search area, but target element $T_{i}$ is not, is $Q(X)-$ $Q\left(X \cup\left\{T_{i}\right\}\right)$. Applying this recursively we can rewrite $P(X$ in search area) in terms of $Q$ as

$$
\begin{array}{r}
P(X \text { in search area })= \\
\sum_{i=0}^{|T \backslash X|} \sum_{\substack{Y \subseteq(T \backslash X), \\
\text { s.t. }|Y|=i}} \begin{cases}Q(X \cup Y), & i \text { is even } \\
-Q(X \cup Y), & i \text { is odd }\end{cases}
\end{array}
$$

Unlike $P\left(X_{k}\right.$ in search area $)$, which also depends on the total number of target elements in the search area, the value of $Q\left(X_{k}\right)$ does not change when the number of target elements in $T \backslash X_{k}$ changes. If we write (10) in terms of $Q$, we can disregard the broken target elements in $X_{j}$, reducing the number of subsets to take into account. The probability that a target element $T_{i}$ is killed is then

$$
\begin{aligned}
& P\left(X_{j} \rightarrow\left(X_{j} \cup\left\{T_{i}\right\}\right)\right)= \\
& \sum_{\substack{X_{k} \subseteq T, \\
\text { s.t. }}} P\left(X_{i} \in X_{k} \text { in search area }\right) P\left(1 /\left|X_{k} \backslash X_{j}\right| \text { killed }\right)= \\
& \sum_{\substack{X_{k} \subseteq\left(T \backslash X_{j}\right), \\
\text { s.t. } T_{i} \in X_{k}}} \sum_{i=0}^{\left|T \backslash\left(X_{k} \cup X_{j}\right)\right|} \sum_{\substack{Y \subseteq\left(T \backslash\left(X_{k} \cup X_{j}\right)\right), \\
\text { s.t. }|Y|=i}} \\
& \begin{cases}Q\left(X_{k} \cup Y\right) P\left(1 /\left|X_{k}\right| \text { killed }\right), & i \text { is even } \\
-Q\left(X_{k} \cup Y\right) P\left(1 /\left|X_{k}\right| \text { killed }\right), & i \text { is odd }\end{cases}
\end{aligned}
$$

Since all possible $X_{k} \cup Y$ are equivalent to some value of $X_{k}$ and the value of $P\left(1 /\left|X_{k}\right|\right.$ killed $)$ does not depend on $Y$, the sum can be factorized as

$$
\begin{aligned}
& \left.P\left(X_{j} \rightarrow X_{j} \cup\left\{T_{i}\right\}\right)\right)=\sum_{\substack{X_{k} \subseteq\left(T \backslash X_{j}\right), \\
\text { s.t. } T_{i} \in X_{k}}} Q\left(X_{k}\right) \sum_{i=0}^{\left|X_{k}\right|-1} \\
& \begin{cases}\left(\begin{array}{c}
\left|X_{k}\right|-1 \\
i
\end{array}\right) P\left(1 /\left(\left|X_{k}\right|-i\right) \text { killed }\right), & i \text { is even } \\
-\left(\begin{array}{c}
\left|X_{k}\right|-1 \\
i
\end{array}\right) P\left(1 /\left(\left|X_{k}\right|-i\right) \text { killed }\right), & i \text { is odd }\end{cases} \\
& K(n)=\sum_{i=0}^{n-1} \begin{cases}\left(\begin{array}{c}
n-1 \\
i
\end{array}\right) P(1 /(n-i) \text { killed }), & i \text { is even } \\
-\left(\begin{array}{c}
n-1 \\
i
\end{array}\right) P(1 /(n-i) \text { killed }), & i \text { is odd }\end{cases}
\end{aligned}
$$

We can define a recursive function

$$
\begin{aligned}
& f(X, Y)= \\
& \begin{cases}f\left(X \backslash\left\{T_{i}\right\}, Y\right)+f\left(X \backslash\left\{T_{i}\right\}, Y \cup\left\{T_{i}\right\}\right), & T_{i} \in X \\
Q(Y) K(|Y|), & X=\emptyset\end{cases}
\end{aligned}
$$

which has the property

$$
\begin{aligned}
& f\left(T \backslash\left(X_{j} \cup\left\{T_{i}\right\}\right),\left\{T_{i}\right\}\right)= \\
& \left.\quad \sum_{\substack{X_{k} \subseteq\left(T \backslash X_{j}\right), \\
\text { s.t. } T_{i} \in X_{k}}} Q\left(X_{k}\right) K\left(\left|X_{k}\right|\right)=P\left(X_{j} \rightarrow X_{j} \cup\left\{T_{i}\right\}\right)\right) .
\end{aligned}
$$

We notice that by selecting $X$ and $Y$ appropriately, $f(X, Y)$ gives us the nonzero nondiagonal elements of the state transition matrix. All the relevant values of $K, Q$ and $f(X, Y)$ can be precalculated in order to create each element of the matrix in constant time, as demonstrated in the algorithm in Fig. 4. The overall complexity of this algorithm is $O\left(n^{2} \cdot 2^{n}\right)$. 
Input: $T, n=|T|, \boldsymbol{P}$ (set in search area), $\boldsymbol{P}(1 / m$ killed $)$ for $i=1$ to $n$ do $\left\{\right.$ Precalculate $K(i)$ to table $K_{i}$ \}

$$
\begin{aligned}
& b_{j} \leftarrow \begin{cases}1, & j=1 \\
a_{j}-a_{j-1}, & j=2, \ldots, i-1 \\
-1, & j=i \text { and } i \text { is even } \\
1, & j=i \text { and } i \text { is odd }\end{cases} \\
& K_{i} \leftarrow \sum_{j=1}^{i} b_{j} P(1 /(i-j+1) \text { killed }) \\
& \boldsymbol{a} \leftarrow \boldsymbol{b}
\end{aligned}
$$

end for

for $k=0$ to $n$ do $\left\{\right.$ Precalculate $Q(X)$ to hash table $\left.Q_{X}\right\}$

for all subsets $X$ such that $X \subseteq T$ and $|X|=n-k$ do $Q_{X} \leftarrow P(X$ in search area $)$

$S_{X, 1} \leftarrow P(X$ in search area $)$

for all elements $T_{i}$ such that $T_{i} \in T \backslash X$ do

$$
\begin{aligned}
& \quad Q_{X} \leftarrow Q_{X}+S_{X \cup T_{i}, i} \\
& \quad S_{X, i+1} \leftarrow S_{X, i}+S_{X \cup T_{i}, i} \\
& \text { end for } \\
& \text { end for } \\
& \text { end for }
\end{aligned}
$$

for $k=1$ to $n$ do \{Precalculate $f(X, Y)$ to hash table $\left.f_{X, Y}\right\}$

$$
\begin{aligned}
& \text { for all subsets } Y \text { such that } Y \subseteq T \text { and }|Y|=k \text { do } \\
& X \leftarrow \emptyset \\
& f_{\emptyset, Y} \leftarrow Q_{Y} \cdot K_{|Y|} \\
& \text { Move the last element of } Y \text { from } Y \text { to } X \\
& \text { for } j=2 \text { to } k \text { do } \\
& \text { Move the last element of } Y \text { from } Y \text { to } X \\
& \text { for } i=1 \text { to } j \text { do } \\
& \quad X_{m} \leftarrow \begin{cases}X_{1}, \quad i>1 \\
X_{2}, \quad i=1\end{cases} \\
& \quad \text { Move element } X_{i} \text { from } X \text { to } Y \\
& \quad f_{X, Y} \leftarrow f_{X \backslash\left\{X_{m}\right\}, Y}+f_{X \backslash\left\{X_{m}\right\}, Y \cup\left\{X_{m}\right\}} \\
& \quad \text { Move element } X_{i} \text { back from } Y \text { to } X \\
& \text { end for } \\
& \text { end for } \\
& \text { end for }
\end{aligned}
$$

\section{end for}

\{Construct the state transition matrix $\boldsymbol{A}=$ $\left.\left[a_{m j}\right]_{1 \leq m, j \leq 2^{n}}\right\}$

$\boldsymbol{A} \leftarrow 0_{2^{n}, 2^{n}}$

for all subsets $X_{j}$ such that $X_{j} \subseteq T$ do

$$
a_{j j} \leftarrow 1
$$

for all elements $T_{i}$ such that $T_{i} \in\left(T \backslash X_{j}\right)$ do

Get row number $m$ such that $X_{m}=X_{j} \cup\left\{T_{i}\right\}$

$$
\begin{aligned}
& a_{m j} \leftarrow f_{T \backslash\left(X_{j} \cup\left\{T_{i}\right\}\right),\left\{T_{i}\right\}} \\
& a_{j j} \leftarrow a_{j j}-a_{m j}
\end{aligned}
$$

$$
\text { end for }
$$

\section{end for}

\section{return $A$}

Figure 4. Algorithm for creating state transition matrix for a single sensor fuzed submunition.

\subsection{Example}

With two target elements, A and B, we have state vector $v$

$$
\boldsymbol{v}=\left[\begin{array}{c}
P(\emptyset \text { killed }) \\
P(\text { A killed }) \\
P(\text { B killed }) \\
P(\text { AB killed })
\end{array}\right]
$$

and state transition matrix $\boldsymbol{A}$

$$
\boldsymbol{A}=\left[\begin{array}{cccc}
p_{\emptyset \rightarrow \emptyset} & 0 & 0 & 0 \\
p_{\emptyset \rightarrow A} & p_{A \rightarrow A} & 0 & 0 \\
p_{\emptyset \rightarrow B} & 0 & p_{B \rightarrow B} & 0 \\
0 & p_{A \rightarrow A B} & p_{B \rightarrow A B} & 1
\end{array}\right]
$$

where

$$
\begin{aligned}
p_{\emptyset \rightarrow A} & =f(B, A)=f(\emptyset, A)+f(\emptyset, A B) \\
& =Q(A) K(1)+Q(A B) K(2) \\
p_{\emptyset \rightarrow B} & =f(A, B)=f(\emptyset, B)+f(\emptyset, A B) \\
& =Q(B) K(1)+Q(A B) K(2) \\
p_{A \rightarrow A B} & =f(\emptyset, B)=Q(B) K(1) \\
p_{B \rightarrow A B} & =f(\emptyset, A)=Q(A) K(1) \\
p_{\emptyset \rightarrow \emptyset} & =1-p_{\emptyset \rightarrow A}-p_{\emptyset \rightarrow B} \\
p_{A \rightarrow A} & =1-p_{A \rightarrow A B} \\
p_{B \rightarrow B} & =1-p_{B \rightarrow A B}
\end{aligned}
$$

and

$$
\begin{aligned}
Q(A) & =P(A \text { in search area })+P(A B \text { in search area }) \\
Q(B) & =P(B \text { in search area })+P(A B \text { in search area }) \\
Q(A B) & =P(A B \text { in search area }) \\
K(1) & =\left(\begin{array}{l}
0 \\
0
\end{array}\right) P(1 / 1 \text { killed }) \\
K(2) & =\left(\begin{array}{l}
1 \\
0
\end{array}\right) P(1 / 2 \text { killed })-\left(\begin{array}{l}
1 \\
1
\end{array}\right) P(1 / 1 \text { killed })
\end{aligned}
$$

\subsection{Projectile with Two Sensor Fuzed Submu- nitions}

We now consider the case where an artillery projectile contains two sensor fuzed submunitions, which are released at some altitude. The release point is still assumed to follow a bivariate normal distribution. The center of the search areas of each submunition is assumed to be located at given distances from the release point in the direction of fire, since the submunitions will have forward motion before being sufficiently slowed down, as illustrated in Figure 5.

For tube artillery projectiles containing two submunitions, the centers of the search areas of the submunitions are separated by approximately $300 \mathrm{~m}$, according to (Dullum, 2008). If the radius of the search area for each 
submunition is $100 \mathrm{~m}$, the search areas will not overlap. Therefore, in this paper the search areas are assumed to be non-overlapping. The submunitions are also assumed identical.

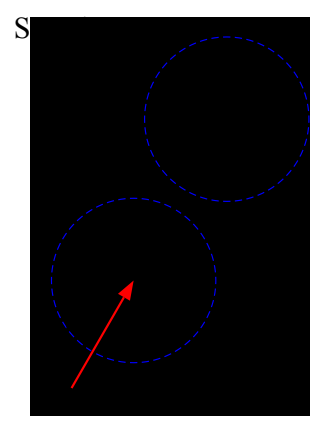

Figure 5. Sensor fuzed munition containing two indentical submunitions, whose non-overlapping search areas are outlined with dashed circles. The release point is at $\mathrm{P}_{0}$, after which submunition 1 travels distance $d_{1}$ and submunition 2 distance $d_{2}$. The direction of fire is represented by the vectors.

The encounter probabilities have to be constructed for both search areas. We denote the subset in the first search area by $S_{1}$ and the subset in the second search area by $S_{2}$, with the constraint that $S_{1} \cap S_{2}=\emptyset$, i.e., the search areas contain disjoint subsets. The probability vector $\boldsymbol{P}$ (sets in search area) can be calculated in a similar manner as in the single submunition case and will have length $3^{n}$. The state transition matrix can be constructed using an approach similar to the single submunition case. Let us redefine the variables as

$$
\begin{gathered}
Q(X, Y)=\sum_{\substack{S_{1} \subseteq T, \\
\text { s.t. } X \subseteq S_{1} \subseteq}} \sum_{S_{2} \subseteq T S_{1},} P\left(S_{1}, S_{2} \text { in search area }\right) \\
P(0 / n \text { killed })= \begin{cases}1-n \cdot P(1 / n \text { killed }), & n>0 \\
1, & n=0\end{cases} \\
K_{0}(n)= \begin{cases}\sum_{i=0}^{n}\left(\begin{array}{l}
n \\
i
\end{array}\right) P(0 /(n-i) \text { killed }), & i \text { is even } \\
-\sum_{i=0}^{n}\left(\begin{array}{c}
n \\
i
\end{array}\right) P(0 /(n-i) \text { killed }), & i \text { is odd }\end{cases}
\end{gathered}
$$

$$
\begin{aligned}
& f_{1}(X, Y, Z)= \\
& \left\{\begin{array}{cc}
f_{1}\left(X \backslash\left\{T_{i}\right\}, Y, Z\right)+f_{1}\left(X \backslash\left\{T_{i}\right\}, Y \cup\left\{T_{i}\right\}, Z\right) & \\
\quad+f_{1}\left(X \backslash\left\{T_{i}\right\}, Y, Z \cup\left\{T_{i}\right\}\right), & T_{i} \in X \\
Q(Y, Z) K(|Y|) K_{0}(|Z|) & \\
\quad+Q(Z, Y) K(|Z|) K_{0}(|Y|), & X=\emptyset
\end{array}\right.
\end{aligned}
$$

$$
\begin{aligned}
& f_{2}(X, Y, Z)= \\
& \left\{\begin{array}{cc}
f_{2}\left(X \backslash\left\{T_{i}\right\}, Y, Z\right)+f_{2}\left(X \backslash\left\{T_{i}\right\}, Y \cup\left\{T_{i}\right\}, Z\right) & \\
\quad+f_{2}\left(X \backslash\left\{T_{i}\right\}, Y, Z \cup\left\{T_{i}\right\}\right), & T_{i} \in X \\
(Q(Y, Z)+Q(Z, Y)) K(|Y|) K(|Z|), & X=\emptyset
\end{array}\right.
\end{aligned}
$$

$$
\begin{aligned}
P\left(X_{j} \rightarrow X_{j} \cup\left\{T_{1}\right\}\right)= & f_{1}\left(T \backslash\left(X_{j} \cup\left\{T_{1}\right\}\right),\left\{T_{1}\right\}, \emptyset\right) \\
P\left(X_{j} \rightarrow X_{j} \cup\left\{T_{1}, T_{2}\right\}\right)= & f_{2}\left(T \backslash\left(X_{j} \cup\left\{T_{1}, T_{2}\right\}\right),\right. \\
& \left.\left\{T_{1}\right\},\left\{T_{2}\right\}\right)
\end{aligned}
$$

With the above definitions, $Q, f_{1}$ and $f_{2}$ can be precalculated in the same way as $Q$ and $f$ in Fig. 4. The state transition matrix for two submunitions can be created in time $O\left(n \cdot 3^{n}\right)$, which is the complexity of precalculating $Q(X, Y)$ recursively for all possible $X, Y \in T$.

\section{Failure Probability of the System}

Arbitrary fault logics may be used to determine which broken subsets correspond to a broken system. The probability that the system breaks is the sum of these subset destruction probabilities in $\boldsymbol{v}(k)$,

$$
P(\text { system breaks })=\sum_{\substack{X_{i} \subseteq T, \\ \text { s.t. } g\left(X_{i}\right)=1}} v_{i}(k)
$$

where function $g$ contains the fault logics. The value of $g(X)$ is 1 if $X$ corresponds to a broken system according to the fault logic and 0 otherwise.

The smallest number of projectiles needed to guarantee failure of the system with a given confidence level $\alpha$ can be obtained simply by starting at $k=1$ and calculating $P$ (system breaks) repeatedly while increasing the value of $k$ until $P($ system breaks $) \geq \alpha$.

\section{Conclusions}

The placement of the aimpoint has a significant impact as well as the trajectories of the submunitions, here modeled as offset vectors. The trajectories of the submunitions from expulsion from the carrier projectile to their activation need further study.

We also need to address the target location error and the mean release point error. The mean release point may not necessarily coincide with the actual aimpoint and the aimpoint may also have a systematic error. One approach to handling these errors is to assume that the errors follow a bivariate normal distribution and discretize the region into a number of points and iterating over them.

The method can be generalized to having target elements with different properties, which would translate to different detection, hit and kill probabilities.

\section{Acknowledgment}

The authors would like to thank Ilmari Kangasniemi and Janne Valtonen for their valuable input on the manuscript.

\section{References}

Ove Dullum. Cluster weapons - military utility and alternatives. FFI-rapport 2007/02345, Norwegian Defence Research Establishment (FFI), Norway, 2008.

Marius Halsør and Lars Kvifte. Metoder for effektberegning av smart artilleriammunisjon [Methods for determining 
the effect of smart artillery ammunition]. FFI/RAPPORT2003/00084, Forsvarets Forskningsinstitutt (Norwegian Defence Research Establishment), 2003.

Jyri Kosola and Tero Solante. Digitaalinen taistelukenttä - informaatioajan sotakoneen tekniikka [Digital Battlefield], volume 35 of Julkaisusarja 1. Maanpuolustuskorkeakoulu, Sotatekniikan laitos, Helsinki, 3rd edition, 2013. ISBN 978951-25-2503-4.

NATO Standardization Office. STANAG 4654: Indirect fire appreciation modelling. Standardization agreement, NATO Standardization Office, Brussels, 2012.

Ville Pettersson, Eric Malmi, Sampo Syrjänen, Bernt Åkesson, Tapio Heininen, and Esa Lappi. Simulating the effect of indirect fire on a multiple target element system. In Sergey Repin, Timo Tiihonen, and Tero Tuovinen, editors, Proceedings of CAO2011: ECCOMAS thematic conference on computational analysis and optimization, volume No. A 1/2011 of Reports of the Department of Mathematical Information Technology, Series A. Collections, Jyväskylä, Finland, June 9-11 2011. University of Jyväskylä. ISBN 978-951-39-43318. 